Journal of Applied Fluid Mechanics, Vol. 14, No. 6, pp. 1657-1668, 2021. Available online at www.jafmonline.net, ISSN 1735-3572, EISSN 1735-3645. https://doi.org/10.47176/jafm.14.06.32319

\title{
Wall Shear Stress-Based Hemodynamic Descriptors in the Abdominal Aorta Bifurcation: Analysis of a Case Study
}

\author{
A. A. Soares ${ }^{1,2 \dagger}$, F. A. Carvalho ${ }^{1}$ and A. Leite Le $^{1,3}$ \\ ${ }^{1}$ ECT/UTAD - School of Science and Technology, University of Trás-os-Montes e Alto Douro, Vila \\ Real, 5000-801, Portugal \\ ${ }^{2}$ CIENER-INEGI/LAETA - Institute of Science and Innovation in Mechanical and Industrial \\ Engineering, Porto, Portugal \\ ${ }^{3}$ C-BER - INESC TEC - Center for Biomedical Engineering Research, Porto, Portugal \\ $†$ Corresponding Author Email: asoares@utad.pt
}

(Received October 25, 2020; accepted May 18, 2021)

\begin{abstract}
The knowledge of hemodynamic behaviour in the abdominal aorta artery bifurcation is of great importance for the early diagnosis of several cardiovascular diseases common in this bifurcation. The work developed focuses on a case study of hemodynamic in the abdominal aorta artery bifurcation, based on a realistic 3D geometric model reconstructed from 2D medical images of a real patient. Hemodynamic quantities based on the wall shear stress (WSS) of the abdominal aorta bifurcation are analysed and is presented an alternative analysis of the well-established stress hemodynamic descriptors to identify specific zones of the artery with a higher probability of developing cardiovascular diseases. The individual analysis of different zones of the artery allowed to obtain information that can remain masked when whole artery is considered as a single zone. The reported results provide a correlation between the analysed stress hemodynamic descriptors and the area of the wall artery. Then, the aim of this work is the identification of regions at the luminal surface subject to atherosusceptible WSS phenotypes. For the patient studied, the analysis presented allowed the identification of the patient's propensity to develop atherosclerosis, according to the hemodynamic descriptors time-averaged WSS (TAWSS), oscillatory shear index (OSI), and relative residence time (RRT). Thus, this work offers a new way of looking to the stress hemodynamic descriptors.
\end{abstract}

Keywords: CFD; Hemodynamic descriptors; Cardiovascular diseases; Doppler ultrasonography; Oscillatory shear index.

\section{NOMENCLATURE}

$\begin{array}{ll}\text { TAWSS } & \text { Time-Average Wall Shear Stress } \\ \text { TAWSSG } & \text { Time-Average Wall Shear Stress Gradient } \\ \text { TAWSSV } & \text { Time-Average Wall Shear Stress Vector } \\ \text { OSI } & \text { Oscillatory Shear Index } \\ \text { WSS } & \text { Wall Shear Stress } \\ \text { WSSG } & \text { Wall Shear Stress Gradient } \\ \rho & \text { blood density } \\ \varepsilon & \text { fraction of erythrocytes }\end{array}$

$\begin{array}{ll}\rho_{p} & \text { plasma density } \\ \rho_{\varepsilon} & \text { erythrocytes density } \\ p & \text { pressure } \\ t & \text { time } \\ T & \text { cycle cardiac period } \\ u & \text { velocity } \\ \mu & \text { dynamic viscosity }\end{array}$

\section{INTRODUCTION}

The maintenance of cardiovascular health, as well as the initialization and location of different cardiovascular diseases is extremely important and can be strongly influenced by shear stresses produced by blood flow in the walls of the arteries (Caballero and Laín 2015). Several arterial diseases present mainly in zones of irregular geometry result from the combination of biomechanical processes that take place in the walls of the vessels at the cellular level and of hemodynamic factors that result from the interaction of blood flow with the vessel walls (Mesri et al. 2017; Ong et al. 2019).

It is well known that the WSS expresses the deceleration of blood flow near the artery wall, which 
results of the tangential frictional force exerted on it, (Giddens et al. 1993; Soares et al. 2011). Several studies have shown that the development of cardiovascular diseases, namely the appearance of atherosclerotic plaques, is related to low WSS values and high temporal oscillations in their direction, (Chatzizisis et al. 2008; Ku et al. 1985).

On the other hand, computational fluids dynamics has been applied with promising results to Biomedical engineering problems (Taylor 1996; Baumann et al. 2020). In complex geometries, the combination of high WSS and positive wall shear stress gradient (WSSG) can to trigger aneurysm initiation and the understanding of the response of the endothelial cells to the high WSS combined with WSSG can aid to improve strategies to deal with disease (Dolan et al. 2013). However, there is no consensus among researchers about this subject (Peiffer et al. 2013; Xing et al. 2018). Thus, further research is needed to elucidate about the spatial and temporal variations of the WSS. Although the correlation between WSS and plaque location is not fully understood, several parameters that are derived from the WSS distribution, called hemodynamic descriptors, have been identified as possible indicators for sites susceptible to atherosclerotic lesions (Taylor et al. 1998; Knight et al. 2010; Ameenuddin and Anand 2020). In addition to the spatial distribution of flow parameters, its temporal variation during the cardiac cycle has also been proposed as a decisive atherogenic factor since the complex configuration of the abdominal aorta bifurcation promotes temporal oscillations of the WSS during the cardiac cycle (Wen et al. 2010). Low TAWSS, high OSI, high RRT and high concentration of low-density lipoprotein are observed in the superior mesenteric artery and below the renal bifurcations, in an idealized abdominal aorta (Ameenuddin and Anand 2020). However, WSS is a consequence of the direct interaction of blood flow with the wall artery and therefore, individual variations in the arterial anatomy influence the hemodynamic behaviour (Soares et al. 2017).

Malek et al. (1999) state that normal values for wall shear stresses, which do not cause damage to endothelial cells, are in the range of 1 to $7 \mathrm{~Pa}$ and when the wall is subjected to low WSS below $1 \mathrm{~Pa}$, are susceptible to the development of atherosclerotic plaques. The second situation is common in artery walls where there is greater geometric complexity by being subject to deceleration and blood recirculation. On the other hand, stresses between 7 and $34 \mathrm{~Pa}$ leads to the tendency of thrombus formation and stresses higher than $34 \mathrm{~Pa}$ leads to the endothelium degradation by mechanical wear (Ortiz et al. 2007). Thus, it can be stated that the shear stresses in the walls can induce transformations in the endothelial cells that favour the development of atherosclerosis, predominantly in low and oscillatory WSS regions, while the regions of vasculature exposed to a physiological shear stress are protected (Chatzizisis et al. 2008; Cheng et al. 2006; Cecchi et al. 2011).

From a practical point of view this type of work can contribute to a better understanding of some biomedical problems, such as, for example, taken into account the implications of WSS in atherosclerosis, the hemodynamic in the vascular structures can be investigated based on the localized WSS regions (Taylor 1996). Note that the WSS quantification in the aorta artery can help reveal how WSS influence atherogenesis (Boyd et al. 2016; Mohamied et al. 2014). The WSS analysis can also be extended to obtain functional information of cardiovascular flow (Wong et al. 2017), to study stents (Benndorf et al. 2009) and heart valves (Haggag 1990).

Despite computational fluid dynamics to be a consolidated tool to study hemodynamic quantities such as WSS, the relation of these quantities with cardiovascular diseases still not well consolidated. Then, this paper aims contribute to a better understanding of those relations through an alternative analysis of some hemodynamic quantities. In this analysis, the hemodynamic descriptors are related with the propensity to develop cardiovascular diseases in different zones of the arterial model. The area of these zones was estimated by the number of nodes of the computational mesh on the artery wall. The main advantage of this analysis is to allow discriminate different artery zones by its area and by the range of values of the hemodynamic descriptors, becoming clearer some information, which sometimes stays masked in an analysis more global.

\section{Methods}

\subsection{Geometry reconstruction}

The reconstruction of the abdominal aorta bifurcation geometry was done through the segmentation of 66 medical images with contrast and a thickness of $1.5 \mathrm{~mm}$, obtained by computational tomography. The images were manually segmented, delineating the artery boundaries present in each of them.

After the treatment of all images, the 3D model of the geometry of the artery was generated. To improve the model, namely with respect to surface smoothing, it was imported into MeshLab where a Laplace filter with 15 iterations was applied, resulting in a more homogeneous surface, Fig. 1.

\subsection{Blood properties}

The dynamic viscosity and density of the blood was quantified taken into account that the plasma at $37^{\circ} \mathrm{C}$ has a viscosity of $1.32 \mathrm{mPa} . \mathrm{s}$ and density $1025 \mathrm{~kg} / \mathrm{m}^{3}$, and the erythrocyte density is $1100 \mathrm{~kg} / \mathrm{m}^{3}$ (Burton 1965; Caro et al. 1971; Lowe et al. 1993).

The fraction of erythrocytes $(\varepsilon=0.387)$ was obtained from the patient blood tests. Then, the blood density $(\rho)$ was determined by the weighted average of the plasma density $\left(\rho_{p}\right)$ with erythrocytes density $\left(\rho_{\varepsilon}\right)$, obtaining the value of $1054.025 \mathrm{~kg} / \mathrm{m}^{3}$,

$\rho=\rho_{p}(1-\varepsilon)+\rho_{\varepsilon}(\varepsilon)$

Despite the blood rheology impact in the NavierStokes equations, its influence is not relevant in large vessels (Box et al. 2005). Thus, in this work a 


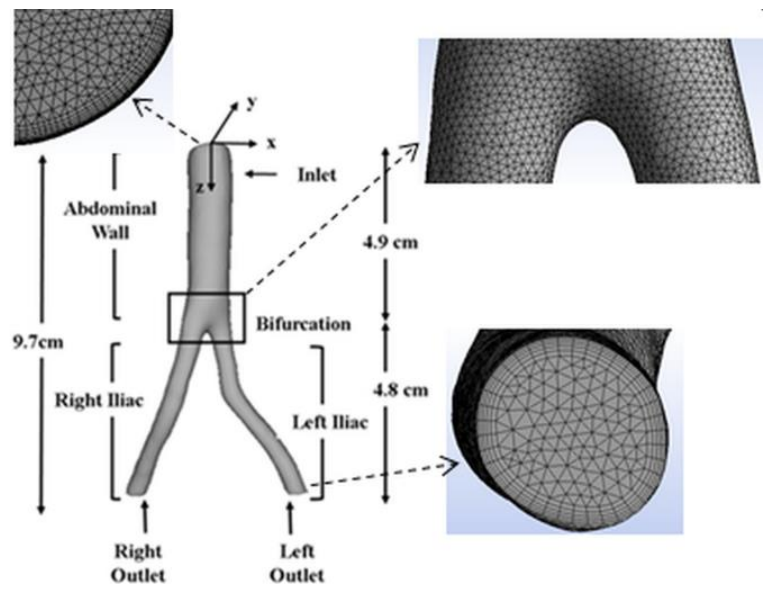

Fig. 1. Model of the abdominal aorta bifurcation with identification of the study zones and Mesh views.

Newtonian viscosity model was used. Among the several Newtonian models for viscosity, available in literature, the model proposed by Lee and Steinman (2007) was used,

$$
\mu=\left(1.4175+5.878 \varepsilon-15.98 \varepsilon^{2}+31.964 \varepsilon^{3}\right) \mu_{p}
$$

where $\mu$ is the dynamic viscosity of blood and $\mu_{p}$ is the plasma viscosity. Our choice fell on this model since it allows to use the clinical data of the patient. Thus, the patient viscosity is $4.1602 \mathrm{mPa} . \mathrm{s}$. The patient is a 50 years old woman.

\subsection{Governing equations}

The blood is considered an incompressible fluid with Newtonian behaviour. The artery wall is rigid and impermeable. The number of Reynolds is always less than 1000, in whole artery. Thus, the governing equations (Bird et al. 2007; Caballero and Laín 2015) for the blood flow in the abdominal aorta bifurcation are given by

$$
\begin{aligned}
& \nabla \cdot u=0 \\
& \rho\left[\frac{\partial u}{\partial t}+(u \cdot \nabla) u\right]=-\nabla p+\mu \nabla^{2} u,
\end{aligned}
$$

where $u$ represents the velocity $(\mathrm{m} / \mathrm{s}), \rho$ the blood density $\left(\mathrm{kg} / \mathrm{m}^{3}\right), p$ the pressure $(\mathrm{Pa})$ and $\mu$ the dynamic viscosity (Pa.s).

\subsection{Boundary conditions}

The velocity waveform at the inlet of the abdominal aorta was measured experimentally by pulsed Doppler ultrasonography at the inlet of the abdominal aorta artery (Fig. 1).

The time-dependent inlet velocity waveform was obtained from the adjustment of the Fourier series to the experimental waveform of four heart beats measured by Doppler ultrasonography. The blood flow rate of the patient is shown in Fig. 2, for one cycle cardiac with a period of $0.63 \mathrm{~s}$. At the inlet boundary, a velocity waveform with a parabolic shape was imposed. Although parabolic profiles are often used in this type of studies, they may display significantly different peak and radial velocities

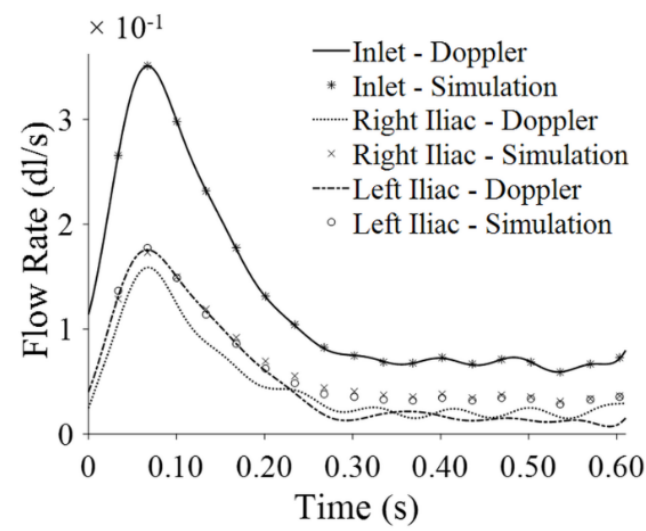

Fig. 2. Comparison of the flow rates at the inlet of the abdominal aorta artery and at the iliac outlets, obtained by Doppler ultrasonography (lines) and simulation (symbols).

when compared with real profiles and therefore may change velocity patterns and produce inaccurate hemodynamic assessments (Youssefi et al. 2018; Soares et al. 2021).

The time step used in the simulations was $0.0335 \mathrm{~s}$. Preliminary tests carried out using a time step two and four times smaller showed that the gain in accuracy of the WSS which resulted from the use of the smallest step time was overall $<2 \%$. Weighing those marginal differences against the disproportionately large increase in CPU time, the $0.0335 \mathrm{~s}$ step time was considered adequate for the present study since it provided sufficient numerical resolution.

In the left and right iliac outlets a pressure of $100 \mathrm{mmHg}$ was imposed, since the systole pressure of a healthy human is around $120 \mathrm{mmHg}$ and diastolic pressure is $80 \mathrm{mmHg}$. Once the pressure waveform at both outlets is the same, the flow distribution will be dictated by the outlet area. Considering that the walls of the artery are rigid, they do not react to the pressure variations then for a given inlet blood flow velocity waveform, the same 
relative pressure field is always obtained regardless of the pressure value imposed at the iliac outlets, Fig. 1. On the other hand, wall properties as a compliant vessel wall does not significantly influence hemodynamics but does significantly increase computational time. (Parker et al. 2019). Then, the inner walls of the artery are assumed to be rigid and subject to the no-slip condition.

\subsection{Numerical method}

The computational simulations presented in this work were performed on Fluent software. The governing equations were solved numerically using the finite volume method. To discretize the momentum equations, we used the second-order upwind scheme, the second-order scheme for the interpolation of the pressure and for the gradient discretization was used the scheme based on GreenGauss node. For the coupling between pressure and velocity the Semi-Implicit Method for Pressure Linked Equations (SIMPLE) algorithm was used. We chose this algorithm because it is easy to implement and is suitable for the problem addressed (Caballero and Laín 2015). Governing equations are integrated for each of the control volumes, resulting in a system of algebraic equations for the discrete dependent variables that are solved numerically until the convergence criterion is reached. The simulations were considered convergent when both the residues of the continuity and momentum equations are less than $10^{-6}$ (Caballero and Laín 2015). For choice of the appropriate mesh, additional tests were carried out for the largest value of the blood velocity. These tests were performed comparing three meshes with $\sim 5 \times 10^{4}, 10^{5}$ and $2 \times 10^{5}$ nodes, all of them structured near of the wall composed by prismatic elements and unstructured in the central zones of the artery composed by tetrahedral elements. The prismatic elements in the near of the wall regions due to its high geometric anisotropy, when subjected to high aspect ratios, maintain the geometric quality. Then all meshes are structured and more refined near of the walls. The inflation technique was used to setup the growth of six inflation layers from the surface of the boundary, in order to capture the boundary layer effects. The mesh is inflated in the direction normal to the boundary until it completely covers the thickness of the boundary layer. No slip boundary condition in the artery wall imposes a non-linear decrease of the blood velocity up to a point at the wall where the blood has zero velocity. Then we need to have in these regions a sufficiently fine mesh to adequately capture the rapid change in the velocity. Thus the inflation layers are a critical component to create a good mesh for CFD because it is important to capture the velocity gradients correctly. This technique ensures that fine resolution is achieved near of the wall, Fig. 1. Furthermore, it results in a smooth transition between the structured and unstructured cells and decreases the overall cell number. The results showed that the gain in accuracy when the mesh with $\sim 2 \times 10^{5}$ nodes is used, comparing with the mesh with $\sim 5 \times 10^{4}$ nodes, was less than $3 \%$. Therefore, the mesh with $\sim 10^{5}$ nodes was considered adequate since they provided sufficient numerical resolution. The results were processed after a cardiac cycle, since preliminary studies for WSS after two and three cardiac cycles did not show significant differences, less than $1 \%$.

\subsection{Stress hemodynamic descriptors}

To facilitate the analysis of the results of the case study investigated, an overview is presented about stress hemodynamic descriptors and their correlation with some diseases of the artery walls. The most commonly used WSS-based descriptors to characterize the hemodynamic of the abdominal aorta artery bifurcation are the time-average wall shear stress (TAWSS), time-average wall shear stress vector (TAWSSV), oscillatory shear index (OSI) and the time-average wall shear stress gradient (TAWSSG) (Knight et al. 2010).

The TAWSS descriptor is the magnitude of the total shear stress applied on the walls obtained by integrating, for each node, the WSS vector magnitude, along the cardiac cycle.

TAWSS $=\frac{1}{T} \int_{0}^{T}|\overrightarrow{\mathrm{WSS}}| d t$

where $T$ represents the period (s) corresponding to a cardiac cycle and |WSS| the instantaneous magnitude of the WSS $(\mathrm{Pa})$.

According $\mathrm{Ku}$ et al. (1985), very low values of TAWSS, less than $0.4 \mathrm{~Pa}$, are the main factor that favours the arises of atherosclerosis. TAWSS values between 1.5 and $7.5 \mathrm{~Pa}$ promote the maintenance of normality in the artery, while higher values promote the development of thrombosis in this region and are present in the convex parts of the curved regions of the artery (Mohamied et al. 2015; Morbiducci et al. 2010). Even higher values of this parameter, greater than $15 \mathrm{~Pa}$, may even lead to the rupture of red blood cells and consequent degradation of the endothelium (Lee et al. 2009), Fig. 3a).

The TAWSSV $(\mathrm{Pa})$ is defined as

$\operatorname{TAWSSV}=\frac{1}{T}\left|\int_{0}^{T} \overrightarrow{\mathrm{WSS}} d t\right|$

The OSI, which is a dimensionless descriptor, allows the identification of zones of the artery wall that are subject to large variations of the WSS, $i . e$. it reveals the general oscillation of the WSS vector during the cardiac cycle and is given by

$\mathrm{OSI}=0.5\left(1-\frac{\left|\int_{0}^{T} \overrightarrow{\mathrm{WSS}} d t\right|}{\int_{0}^{T}|\overrightarrow{\mathrm{WSS}}| d t}\right)$

The OSI identifies locations where the direction of the vector of WSS is different from the predominant direction of blood flow during the cardiac cycle. The values of this descriptor can vary from 0 (WSS vector without variations throughout the cardiac cycle) to $0.5\left(180^{\circ}\right.$ deflection of the direction of the WSS vector). OSI values between 0.1 and 0.3 correspond to favourable conditions for maintenance 
of the healthy artery whereas higher values between 0.3 and 0.5 favour the development of atherosclerosis, inflammation of the artery and internal thickening of the wall artery (Morbiducci et al. 2010; Fytanidis et al. 2014). According to Zeng and $\mathrm{Li}$ (2012), values close to 0.5 occur in zones where these directions present great oscillations during a large part of the cardiac cycle, which suggests a greater potential for the development of atherosclerosis. In the zone of the bifurcation and in places of greater turbulence this descriptor presents high values, and is related with the formation of recirculation zones (Golemati et al. 2007).

On the other hand, for instance O'Rourke (2012) found that, for a studied patient, thrombus growth was observed at regions of low oscillatory shear index (OSI <0.1). Arzani et al. (2014) found a strong correlation between near-wall recirculation regions (low OSI) and thrombus accumulation, Fig. $3 b$ ). Thrombus formation is initiated by the platelets activation. This complex process is dynamic and determined by the interaction of biochemical and biomechanical factors which include high shear stress and exposure time to such stresses. Note that currently there is no consensus among researchers about the threshold values to identify thrombogenic risk and atheroprone/ atheroprotective WSS-based hemodynamic descriptor (Peiffer et al. 2013). Then, in this study, the adopted threshold values may not agree with those proposed by other authors (Gallo et al. 2018).
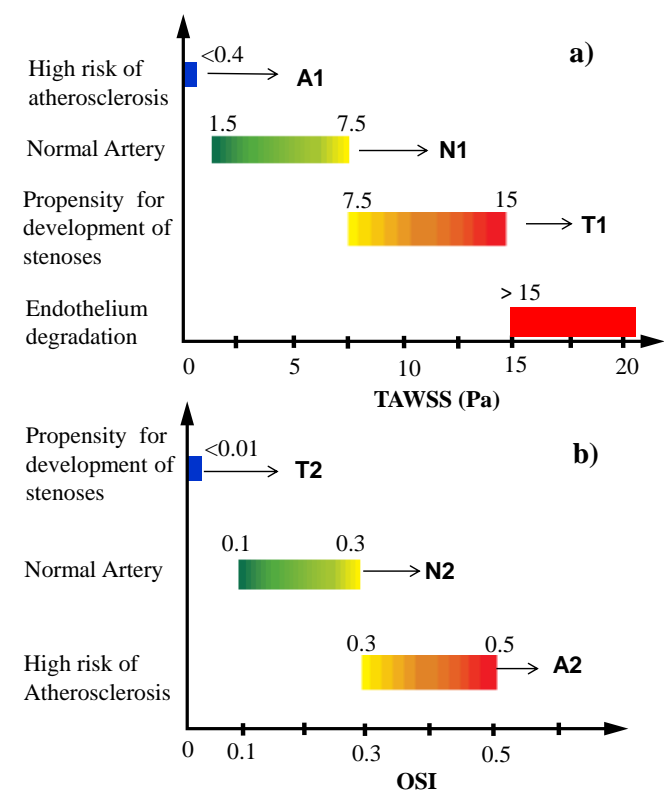

Fig. 3. Association of some pathologies with a) TAWSS and b) OSI. N1 and N2 are the range values for a healthy artery, $\mathrm{A} 1$ and $\mathrm{A2}$ are the range values with risk of atherosclerosis and $\mathrm{T} 1$ and $\mathrm{T} 2$ are the range values with risk of thrombosis, for TAWSS and OSI descriptors, respectively.

On the other hand, the time-average wall shear stress gradient $(\mathrm{Pa} / \mathrm{m})$, is an important temporal descriptor in the modulation of gene expression in endothelial cells. These genes regulate proliferation, migration, and apoptosis in cells. The time-average wall shear stress gradient is given by

TAWSSG $=\frac{1}{T} \int_{0}^{T}(\mathrm{WSSG}) d t$

where

$$
\mathrm{WSSG}=\sqrt{\left(\frac{\partial \mathrm{WSS}_{\mathrm{x}}}{\partial x}\right)^{2}+\left(\frac{\partial \mathrm{WSS}_{\mathrm{y}}}{\partial y}\right)^{2}+\left(\frac{\partial \mathrm{WSS}_{\mathrm{z}}}{\partial z}\right)^{2}}
$$

is the wall shear stress gradient (WSSG), which describes the spatial change of the WSS at a point in time (Soulis et al. 2014) and can therefore be considered an endothelial cell stress marker (Lee et al. 2009). The WSSG is widely used to study hemodynamic in aneurysms, indicates the spatial variation of shear stress in the normal and tangential directions of the artery walls. High WSSG values generally are obtained at recirculation locations.

\section{RESULTS AND DISCUSSION}

\subsection{Verification}

Figure 2 shows the comparison between the flow rates waveform obtained with the adjustment of Fourier series to the experimental Doppler data and the flow rate obtained from the computational simulations. The experimental velocity waveform at the abdominal inlet and at the two iliac outlet branches were obtained through Doppler ultrasound data collected at $\sim 4.8 \mathrm{~cm}$ from the bifurcation of the abdominal aorta artery, Fig. 1.

From Fig. 2 we can see that flow in the left and right iliac outlets, in experimental Doppler measurements, present significant differences between them and such differences can be explained by the anatomical variations since there is no symmetry between the iliac branches. Thus, it can be inferred that for another patient, your arterial geometry will be different from the geometry under study, then the results should be different, even for simulations under same conditions.

From the analysis of Fig. 2, it is also verified that, in Doppler measurements, the mass conservation equation is not satisfied since the inlet flow rate is not equal to the output flow rate. This can be explained by the experimental error associated with the Doppler velocity measurements and the error associated with the Fourier series adjustment to the velocity waveform. The estimation of the experimental error, during the cardiac cycle, was made through the relative percentage difference between the flow rate at the inlet and at the outlets. These differences vary between $4.84 \%$ and $24.55 \%$. For example, the inlet velocity measured with the Doppler is maximum at $t=0.0670 \mathrm{~s}$ and the flow rate is $0.3514 \mathrm{dl} / \mathrm{s}$ while at the iliac outlets, at the same time, the flow rate is $0.3344 \mathrm{dl} / \mathrm{s}$, which corresponds to the experimental error $4.84 \%$. However, for $t=0.315 \mathrm{~s}$, corresponding to the half cardiac cycle, 
when the velocity is practically constant, the flow rate at the inlet is $7.487 \times 10^{-2} \mathrm{dl} / \mathrm{s}$ and at the outlets is $5.649 \times 10^{-2} \mathrm{dl} / \mathrm{s}$ then the error is $24.55 \%$ which correspond to the maximum value throughout the cardiac cycle. Thus, according to this evaluation, the mean experimental error associated with Doppler measurements over the cycle was estimated in $\sim 17.6 \%$.

From the comparison between the flow rate obtained with the Doppler measurements and the computational simulations, the maximum difference between them for the outlet of right iliac occurs at $t=0.1340 \mathrm{~s}$ and is $3.163 \times 10^{-2} \mathrm{dl} / \mathrm{s}$, corresponding to a difference in velocity of $0.11 \mathrm{~m} / \mathrm{s}$. For the left outlet is $1.095 \times 10^{-2} \mathrm{dl} / \mathrm{s}$, which corresponds to a velocity difference of $0.04 \mathrm{~m} / \mathrm{s}$ and occurs at $t=0.2345 \mathrm{~s}$. These differences can be explained by the experimental error associated with Doppler measurements, the Doppler spatial resolution, the differences between Doppler measurements and Fourier series approximations, and the measurements inlet and outlets locations may not coincide with the locations in the computational model of the artery (Soares et al. 2017). On the other hand, the Doppler measurements have not been performed simultaneously so there is no guarantee that the cardiac pulsations correspond to the patient's identical anxiety states. On addition, we cannot ignore the numerical errors characteristics of computational simulations (e.g. mesh resolution and linearization of the governing equations). Note that this case study deals with one patient with a healthy aorta and with 95 heartbeats per minute, maybe due to the patient's anxiety, which is not the normal state of this patient at rest.

\subsection{Distribution of wall shear stress (WSS)}

Figure 4 shows the distribution of wall shear stress for five instants of the heartbeat. In all instants, the higher values of WSS are verified in the walls of the iliac arteries close to the abdominal bifurcation (see the enlarged images). The curved zone in the left iliac also has higher WSS values than in the surrounding regions.

By analysis of Fig. 4 and comparison with the work of Malek et al. (1999), the results suggest that during systole the values of WSS in the specific zone where the bifurcation occurs are higher than $7 \mathrm{~Pa}$, indicating that it is a zone of high stresses, subject to thrombosis. At $t=0.0670 \mathrm{~s}$, the WSS maximum value is $26.8 \mathrm{~Pa}$, it is a worrisome zone because it can, under certain conditions, reach values that can lead to degradation of the endothelium. On the other hand, during the diastole, in the zone corresponding to the abdominal aorta, WSS values below $0.4 \mathrm{~Pa}$ are obtained, which may contribute to the appearance of atherosclerosis.

\subsection{TAWSS, TAWSSV, OSI and TAWSSG descriptors}

Figure 5 shows the distribution of TAWSS, TAWSSV, OSI and TAWSSG over whole artery wall.
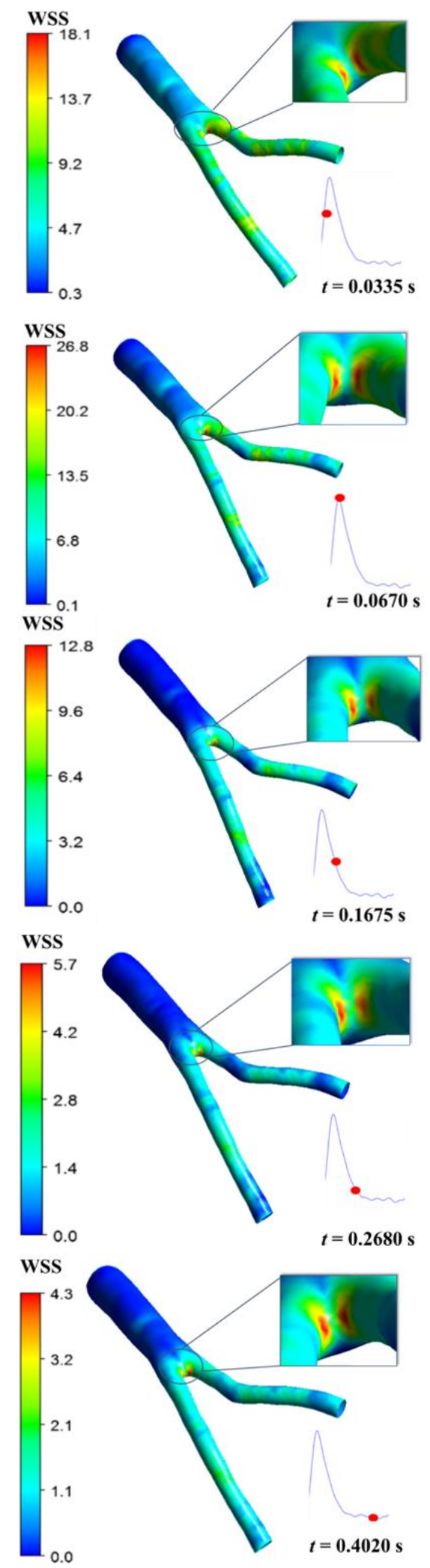

Fig. 4. Distribution of the WSS (Pa) along the abdominal aorta artery.

As can be seen, and as might be expected, the differences of $\sim 0.17 \%$, which is in line with the results obtained by Fytanidis et al. (2014) for a patient-specific but for the aortic arch. In general, in 
the upstream bifurcation zone the values of these descriptors are low and increase slightly towards the iliac. By analysis of Fig. 5 and comparison with Fig. $3 a)$, the results also suggest that the TAWSS values in the specific zone where the bifurcation occurs are greater than $7.5 \mathrm{~Pa}$, which indicates that this zone is subject to formation of thrombus.

Moreover, in the zone corresponding to the abdominal aorta are obtained TAWSS values below $0.4 \mathrm{~Pa}$, which corresponds to conditions of high risk of atherosclerosis.

The OSI appears to have, in general, an inverse behaviour to that observed for the TAWSS and TAWSSV descriptors, that is, the OSI is practically constant along the iliac arteries and the most significant variations occur in the abdominal wall and at the bifurcation. The zones corresponding to low TAWSS values present high OSI values. This observation is consistent with other studies (Youssefi et al. 2018; Lee et al. 2009; Morbiducci et al. 2011). Comparing the distribution of OSI in Fig. 5 with Fig. $3 b$ ), there are three zones with OSI values above 0.3 , which are prone to the occurrence of endothelial dysfunction and, consequently, atherosclerosis $(\mathrm{Ku}$ et al. 1985; Davies 2009).

The results obtained in this study for the TAWSS and OSI distribution are in accordance with the results obtained by Tang et al. (2006) for patients undergoing physical exercise. That is, the TAWSS values increase and the OSI values decrease from the inlet of the abdominal aorta to the iliac arteries outlets. Note that the studied patient is at rest, but with 95 heartbeats per minute, perhaps due to the patient's anxiety.

The TAWSSG is also presented in Fig. 5, which represents the time-average WSS gradient along the cardiac cycle, for whole artery. This descriptor describes the spatial change of WSS at one point during a cardiac cycle $(\mathrm{Pa} / \mathrm{m})$ and can therefore be considered an endothelial cell stress marker (Lee et al. 2009). The TAWSSG is calculated by integrating the WSSG values, which represents the gradient of the shear stresses on the walls at each instant of the cycle, for whole cardiac cycle.

The distribution of TAWSSG is, in general, homogeneous in the abdominal wall. The mean value of this descriptor along the abdominal aorta artery bifurcation is $67.1 \mathrm{~Pa} / \mathrm{m}$ and its maximum value is $735.0 \mathrm{~Pa} / \mathrm{m}$ which occurs in the bifurcation zone.

The Fig. 5 also suggests that there is some relationship between TAWSS and TAWSSG descriptors.

\subsection{Relationship between TAWSS and OSI descriptors}

To investigate which artery zones are subject to the development of cardiovascular diseases, the TAWSS and OSI descriptors were distributed along the $\mathrm{Z}$ axis of the artery, Fig. 6a-b), with the discrimination of the artery zone (abdominal, bifurcation, right iliac and left iliac, Fig. 1. In addition, this information was also combined with the information collected from Fig. 3 to compare the values obtained for these
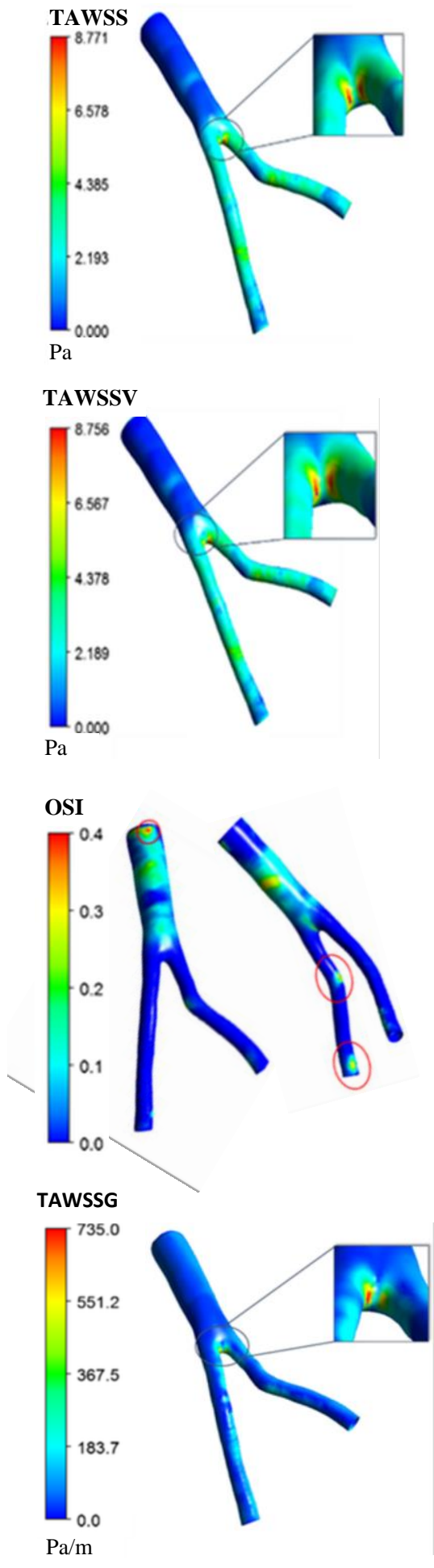

Fig. 5. Distribution of TAWSS, TAWSSV, OSI and AWSSG descriptors over the abdominal aorta artery bifurcation.

descriptors with the possibility of developing cardiovascular diseases. In Fig. 6a-b), N1 and N2 regions correspond to typical TAWSS and OSI values of a healthy artery, $\mathbf{A 1}$ and $\mathbf{A 2}$ correspond to TAWSS and OSI values characteristic of atherosclerosis risk, and $\mathbf{T} 1$ and $\mathbf{T} 2$ correspond to TAWSS and OSI values characteristics of thrombosis risk, respectively. 
Figure 6c) shows the OSI values in function of TAWSS values. In this figure are also delimited seven regions. These regions define the ranges of TAWSS and OSI values that may be associated with the propensity to develop some diseases. Note that the points represented in the Fig. 6 correspond to the nodes in the artery wall of the computational mesh. The results obtained for the relationship between the TAWSS and OSI descriptors in the present study (Fig. 6c) are consistent with the results observed by Fytanidis et al. (2014) for the aortic arch and other studies (Peiffer et al. 2013; Rikhtegar et al. 2012), since the characteristic relation has a similar trend, that is, the OSI values decrease as the TAWSS values increase and vice versa.

The A1A2 region in Fig. 6c) corresponds to the region where there are two simultaneous conditions favourable to the development of atherosclerosis, that is, TAWSS less than $0.4 \mathrm{~Pa}$ and OSI higher than 0.3 . The T1T2 region corresponds to the region where there are also two favourable conditions for the development of thrombosis, that is, TAWSS greater than 7.5 and OSI lower than 0.01 (Fig. 3). Points in the A1A2 region are located only in the abdominal wall and points in region T1T2 belong to the bifurcation. However, it should be noted that N1A2, A1N2 and N1T2 regions only verifies one condition favorable to the development of any of these two cardiovascular diseases.

The analysis of Fig. 6 allows to retrieve the statistical information from the distribution of TAWSS and OSI descriptors at the likely points of disease development. This statistical information is presented in Table 1.
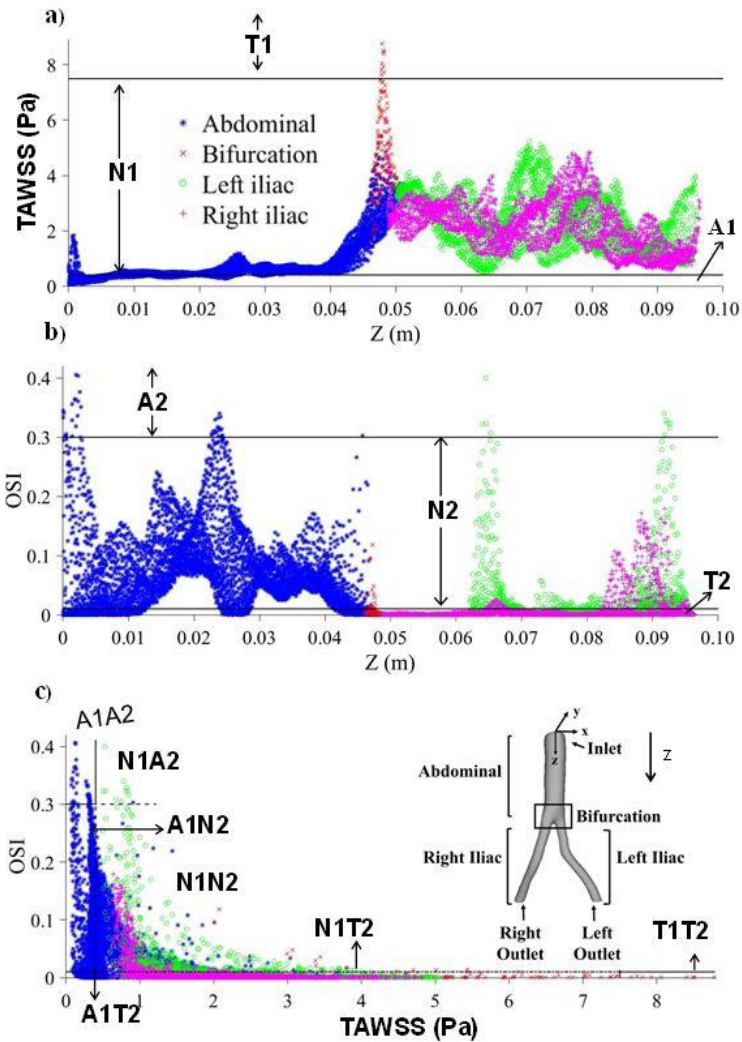

Fig. 6. a) TAWSS and b) OSI along the $Z$ axis and relationship with cardiovascular diseases. $N 1$ and $N 2$ are the range values for a healthy artery, $\mathrm{A1}$ and $\mathrm{A2}$ are the range values with risk of atherosclerosis and $\mathrm{T} 1$ and $\mathrm{T} 2$ are the range values with risk of thrombosis, for TAWSS and OSI descriptors, respectively. c) TAWSS versus OSI and susceptibility to cardiovascular diseases.

Table 1 Percentage (\%) of points in the different zones of the artery, in the regions of Fig. $6 \mathrm{c}$.

\begin{tabular}{|c|c|c|c|c|c|}
\hline \multirow{2}{*}{ Region in Fig. 6c } & \multicolumn{5}{|c|}{ Artery zone } \\
\cline { 2 - 6 } & Abd. & Bif. & Right Iliac & Left Iliac & Total geom. \\
\hline A1A2 & 0.60 & 0 & 0 & 0 & 0.30 \\
\hline N1A2 & 0.02 & 0 & 0 & 0.33 & 0.08 \\
\hline A1N2 & 18.10 & 0 & 0 & 0 & 8.80 \\
\hline N1N2 & 55.94 & 10.06 & 10.95 & 24.03 & 35.82 \\
\hline A1T2 & 6.14 & 0 & 0 & 0 & 2.97 \\
\hline N1T2 & 19.20 & 83.90 & 89.05 & 75.64 & 51.96 \\
\hline T1T2 & 0 & 6.04 & 0 & 0 & 0.07 \\
\hline
\end{tabular}


The seven regions expressed in Table 1 correspond to the regions of Fig. 6c). The points of the wall artery belonging to each region represent the nodes of the computational mesh in the artery wall. Taking into account the homogeneity of the mesh on the artery wall, each node represents, on average, the same area of the artery wall, so we can use the number of nodes as a first approximation of a given area of the wall artery.

Although Fig. 6c) presents some points of the abdominal wall in A1A2 region, which represents the range of values of the descriptors more likely to develop atherosclerosis because they present two conditions favorable to its development, these points correspond only to $0.30 \%$ of the total area of the artery (Table 1).

The A1A2, N1A2, A1N2 and A1T2 regions of Fig. 6c) correspond to those artery regions which exhibit at least one of the conditions favorable to the development of atherosclerosis, i. e., TAWSS or OSI atherosclerotic values. Thus, $12.15 \%$ of the total area of the artery presents one of these conditions and corresponds in Fig. 6a) to $\mathbf{A 1}$ region and in Fig. 6b) to $\mathbf{A} \mathbf{2}$ region. However, as already mentioned, only $0.3 \%$ present both conditions simultaneously (A1A2 region in Fig. 6c). Relative to the abdominal zone, $24.86 \%$ of its area presents one of the conditions, but only $0.60 \%$ presents both conditions.

The A1T2, N1T2 and T1T2 regions of Fig. 6c) correspond to nodes that show TAWSS or OSI values favorable for the development of thrombosis.

In this case, $55 \%$ of the total area of the artery presents at least one of the conditions necessary for the development of this disease, which corresponds in Fig. 6a) to $\mathbf{T} 1$ region and in Fig. 6b) to $\mathbf{T} 2$ region. However, only $0.07 \%$ of the artery area satisfies both conditions simultaneously (T1T2 region). From the overall analysis of Fig. 6 it is seen that a large part of the area of the bifurcation zone has at least one of the favorable conditions for the development of thrombosis. Statistically, this area corresponds to $89.94 \%$ of the area of the wall of the bifurcation. However, only $6.04 \%$ have both conditions.

The N1N2 region of Fig. 6c) corresponds to the artery area that does not present favorable values of TAWSS or OSI for the development of some type of disease. That is, for the artery area free of worries which corresponds to $35.82 \%$ of the total artery area. In Fig. 6b-c) this corresponds to ranges of TAWSS between $1.5 \mathrm{~Pa}$ and $7.5 \mathrm{~Pa}$ and OSI between 0.1 and 0.3 . The A1T2 region corresponds to a region with characteristics for developing all diseases and with a corresponding artery area of $2.97 \%$.

The correlation between TAWSS and OSI (Fig. 6c) was calculated using the Spearman's rank correlation coefficient $(r)$. Spearman's rank coefficient assumes values between -1 and 1 . The value -1 means a perfect negative correlation and 1 means a positive correlation between the two variables. If this coefficient has a value of 0 , the two variables do not depend on each other. The results of the correlation between these two descriptors are represented in Table 2. The results indicate that there is a negative correlation between TAWSS and OSI descriptors, which is in line with the literature for total geometry, e. g. (Fytanidis et al. 2014; Arzani and Shadden 2016).

Table 2 Spearman rank correlation coefficient $r$ between TAWSS and OSI and between the normalized descriptors TAWSS and TAWSSG, for the artery zones. P-values $<0.05$ except the bold value.

\begin{tabular}{|l|c|c|}
\hline \multirow{2}{*}{ Artery zone } & \multicolumn{2}{|c|}{ Descriptors } \\
\cline { 2 - 3 } & $\begin{array}{c}\text { TAWSS and } \\
\text { OSI }\end{array}$ & $\begin{array}{c}\text { TAWSS }_{\text {norm }} \text { and } \\
\text { TAWSSG }_{\text {norm }}\end{array}$ \\
\hline Abdominal & -0.421 & -0.074 \\
\hline Bifurcation & $\mathbf{- 0 . 1 1 2}$ & 0.790 \\
\hline Right Iliac & -0.548 & 0.131 \\
\hline Left Iliac & -0.695 & 0.224 \\
\hline $\begin{array}{l}\text { Total } \\
\text { geometry }\end{array}$ & -0.736 & 0.191 \\
\hline
\end{tabular}

\subsection{Normalized average wall shear stress gradient (TAWSSG) and average wall shear stress (TAWSS)}

Figure 7 shows the relation between the normalized values of TAWSS and TAWSSG along the $\mathrm{Z}$ axis. Both descriptors were normalized to their maximum value of $8.77 \mathrm{~Pa}$ for the TAWSS and $735.0 \mathrm{~Pa} / \mathrm{m}$ for the AWSSG, so that both ranged from 0 to 1 . Since TAWSS and TAWSSG have very different ranges of values, they were normalized to become possible the comparison between them.

The correlation between TAWSS and TAWSSG descriptors seems to be quite significant since the overlap between the values of the two normalized descriptors is very high. Table 2 also shows that for TAWSS and TAWSSG there is a positive correlation for all zones, except in the abdominal artery with $r=$ -0.074 . The $r$ value close to zero indicates that there is no tendency for TAWSS to increase or decrease when TAWSSG increases. It should be noted that in the bifurcation zone the value of the correlation is quite high $(r=0.790)$. From the analysis of Fig. 7, it is inferred that the normalized values of TAWSSG greater than TAWSS occur preferentially in the abdominal zone which is located up to $\mathrm{Z}=0.048 \mathrm{~m}$.

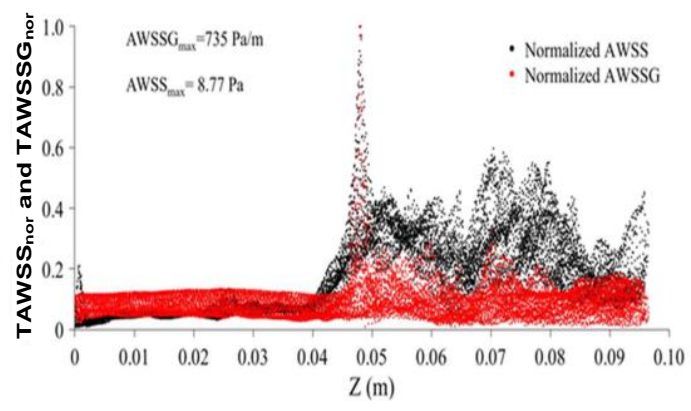

Fig. 7. Distribution of TAWSS and TAWSSG descriptors (normalized) along the $\mathrm{Z}$ axis. 
The bifurcation and iliac zones generally have higher TAWSSnor values than for TAWSSGnor. Statistically, $95.30 \%$ of the points of the bifurcation, $89.93 \%$ of the points of the right iliac and $94.76 \%$ of the points of the left iliac have TAWSSnor greater than TAWSSGnor.

The peak at $\mathrm{Z}=0.048 \mathrm{~m}$ identifies the location of the bifurcation, which is the location where the maximum AWSSG and TAWSS values occur, which is in line with Figs. 4 and 5.

Overall analysis of the data from Figs 5 and 7 shows that $66.81 \%$ of the total artery area is subject to TAWSSnor values greater than TAWSSGnor values.

\section{CONCLUSION}

The main goal of this investigation was the identification of regions at the luminal surface subject to atherosusceptible WSS phenotypes, from threshold values referenced in the literature for the hemodynamic descriptors studied in this work. The investigation is focused in the numerical study of the TAWSS, TAWSSV, OSI, WSSG and TAWSSG hemodynamic descriptors in the abdominal aorta artery bifurcation for a specific-patient and describes relations between them. Under the conditions studied, the analysis presented allowed to identify potential zones of the artery with a higher probability of developing some cardiovascular diseases. Numerous studies have attempted to correlate the development of cardiovascular disease with hemodynamic descriptors values throughout the arterial model. However, this paper presents an alternative analysis which allows to associate different zones of the artery belonging to the same arterial model with some cardiovascular diseases and with the artery wall area where may develop that diseases. Further, for the patient under study the results indicate that the zone most likely to the development of atherosclerosis is the abdominal wall zone and the zone most likely to the development of thrombosis is the bifurcation. However, the percentage of area subject to these conditions is $6.64 \%$, so that may not be enough for the development of the diseases.

To make this assessment more relevant, more quantitative results and comparatives analysis are required for other patients to explore how the relationship between the hemodynamic descriptors found in this investigation, for a specific-patient, may represent a general standard that can be useful in the aid of the therapeutic decision and pharmacological intervention.

\section{REFERENCES}

Ameenuddin, M. and M. Anand (2020). A mixture theory model for blood combined with lowdensity lipoprotein transport to predict early atherosclerosis regions in idealized and patientderived abdominal aorta. Journal of Biomechanical Engineering 142(10). 101008.

Arzani, A. and S. C. Shadden (2016).
Characterizations and Correlations of wall Shear Stress in Aneurysmal Flow. Journal of Biomechanical Engineering 138, 014503-1.

Arzani, A, G. Y. Suh, R .L. Dalman and S .C. Shadden (2014) A longitudinal comparison of hemodynamics and intraluminal thrombus deposition in abdominal aortic aneurysms. American Journal of Physiology-Heart and Circulatory Physiology 307:H1786-H1795.

Baumann, I., M. M. Rashidi, O. Schmid and E. Gutheil (2020). Simulation of patient-specific bi-directional pulsating nasal aerosol dispersion and deposition with clockwise $45^{\circ}$ and $90^{\circ}$ nosepieces. Computers in Biology and Medicine 123, 103816

Benndorf, G., M. Ionescuy, M. V. Alvarado, J. Hipp and R. Metcalfe (2009). Wall shear stress in intracranial self-expanding stents studied using ultra-high-resolution 3D reconstructions. AJNR. American Journal of Neuroradiology 30(3), 479-486.

Bird, R. B., W. E. Stewart and E. N. Lightfoot (2007). Transport Phenomena, Wiley.

Boyd, A. J., D. C. S. Kuhn, R. J. Lozowy and G. P. Kulbisky (2016). Low wall shear stress predominates at sites of abdominal aortic aneurysm rupture, Journal of Vascular Surgery 63(6), 1613-1619.

Box, F. M. A., R. J. Geest, M. C. M. Rutten and J. H. C. Reiber (2005). The Influence of Flow, Vessel Diameter, and Non-Newtonian Blood Viscosity on the Wall Shear Stress in a Carotid Bifurcation Model for Unsteady Flow. Investigative Radiology 40(5), 277-294.

Burton, A. C. (1965). Physiology and biophysics of the circulation. Chicago, IL: Year Book Medical.

Caballero, A. D. and S. Laín (2015). Numerical simulation of non-Newtonian blood flow dynamics in human thoracic aorta. Computer Methods in Biomechanics and Biomedical Engineering 18(11), 1200-1216.

Caro, C. G., J. M. Fitz-Gerald and R. C. Schroter (1971). Atheroma and arterial wall shear. Observation, correlation, and proposal for a shear dependent mass transfer mechanism for atherogenesis. Proceedings of the Royal Society B: Biological Sciences 117, 109-159.

Cecchi, E., C. Gigliol, S. Valente, C. Lazzeri, G. F. Gensini, R. Abbate and L. Mannini (2011). Role of hemodynamic shear stress in cardiovascular disease. Atherosclerosis 214(2), 249-256.

Chatzizisis, Y. S., M. Jonas, A. U. Coskun, R. Beigel, B. V. Stone, C. Maynard, R. G. Gerrity, W. Daley, C. Rogers, E. R. Edelman, C. L. Feldman and P. H. Stone (2008). Prediction of the localization of high-risk coronary atherosclerotic plaques on the basis of low endothelial shear stress: an intravascular ultrasound and histopathology natural history 
study. Circulation 117, 993-1002.

Cheng, C., D. Tempel, R. van Haperen, A. van der Baan, F. Grosveld, M. J. Daemen, R. Krams and R. de Crom (2006). Atherosclerotic lesion size and vulnerability are determined by patterns of fluid shear stress. Circulation 113, 2744-2753.

Davies, P. F. (2009). Hemodynamic shear stress and the endothelium in cardiovascular pathophysiology. Nature Clinical Practice Cardiovascular Medicine 6(1), 16-26.

Dolan, J. M., J. J. Kolega and H. Meng (2013). High Wall shear stress and spatial gradients in vascular pathology: a review. Annals of Biomedical Engineering 41(7), 1411-1427.

Fytanidis, D. K., J. V. Soulis and G. D. Giannoglou (2014). Patient-specific arterial system flow oscillation. Hippokratia 18, 162-165.

Gallo, D., P. B. Bijari, U. Morbiducci, Y. Qiao, Y. J. Xie, M. Etesami, D. Habets, E. G. Lakatta, B. A. Wasserman and D. A. Steinman (2018). Segment-specific associations between local haemodynamic and imaging markers of early atherosclerosis at the carotid artery: an in vivo human study. Journal of the Royal Society Interface 15. 20180352.

Giddens, D. P., C. K. Zarins and S. Glagov (1993). The role of fluid mechanics in the localization and detection of atherosclerosis. Journal of Biomedical Engineering 115(4B), 588-594.

Haggag, Y. A. M. (1990). A comparative study of the shear stress induced in the leakage backflow produced by four types of heart valve prostheses. Proceedings of the Institution of Mechanical Engineers, Part H: Journal of Engineering in Medicine 204 (2), 111-114.

Golemati, S., J. Stoitsis, E. G. Sifakis, T. Balkizas and K. S. Nikita (2007). Using the hough transform to segment ultrasound images of longitudinal and transverse sections of the carotid artery. Ultrasound in Medicine \& Biology. 33, 1981-1932.

Knight, J., U. Olgac, S. Saur, D. Poulikakos, W. Marshall, P. Cattin, H. Alkadhi and V. Kurtcuoglu (2010). Choosing the optimal wall shear parameter for the prediction of 54 plaque location - A patient-specific computational study in human right coronary arteries. Atherosclerosis 21, 445-450.

Ku, D. N., D. P. Giddens, C. K. Zarins and S. Glagov (1985). Pulsatile flow and atherosclerosis in the human carotid bifurcation - positive correlation between plaque location and low and oscillating shear-stress. Arteriosclerosis 5, 293-302.

Lee, S. W., L. Antiga and D. A. Steinman (2009) Correlations among indicators of disturbed flow at the normal carotid bifurcation. Journal of Biomedical Engineering 131: 061013.

Lee, S. W. and D. A. Steinman (2007). On the Relative Importance of Rheology for Image-
Based CFD Models of the Carotid Bifurcation, ASME Journal of Biomechanical Engineering 129(2), 273-278.

Lowe, G., F. Fowkes, J. Dawes, P. Donnan, S. Lennie and E. Housley (1993). Blood viscosity, fibrogen, and activation of coagulation and leukocytes in peripheral arterial disease and the normal population in the Edinburgh artery study. Circulation 87, 1915-1920.

Malek, A. M., S. L. Alper and S. Izumo (199) Hemodynamic shear stress and its role in atherosclerosis. JAMA 282(21), 2035-2042.

Mesri, Y., H. Niazmand and A. Deyranlou (2017). Numerical Study on Fluid-Structure Interaction in a Patient-Specific Abdominal Aortic Aneurysm for Evaluating Wall Heterogeneity and Material Model Effects on its Rupture. Journal of Applied Fluid Mechanics Journal of Applied Fluid Mechanics 10(6), 1699-1709, 2017.

Mohamied, Y., E. M. Rowland, E. L. Bailey, S. J. Sherwin, M. A. Schwartz and P. D. Weinberg (2015). Change of direction in the biomechanics of atherosclerosis. Annals of Biomedical Engineering 43, 16-25.

Morbiducci, U., D. Gallo D., Massai, F. Consolo, L. Antiga, C. Bignardi, A. Redaelli and F. Montevecchi (2011). On the importance of blood rheology for bulk flow in hemodynamic models of the carotid bifurcation. Journal of Biomechanical Engineering 44, 2427-2438.

Morbiducci, U., D. Gallo, D. Massai, F. Consolo, R. Ponzini, L. Antiga, C. Bignardi, M. A. Deriu and A. Redaelli (2010). Outflow conditions for image-based hemodynamic models of the carotid bifurcation: implication for indicators of abnormal flow. Journal of Biomedical Engineering 132: 0910051-09100511.

Ong, C. W., F. Kabinejadian, F. Xiong, Y. R. Wong, M. Toma, Y. N. Nguyen, K. J. Chua, F. S. Cui, P. Ho and H. Leo (2019). Pulsatile Flow Investigation in Development of Thoracic Aortic Aneurysm: An In-Vitro Validated Fluid Structure Interaction Analysis. Journal of Applied Fluid Mechanics 12(6), 1855-1872.

Ortiz, J. P., K. L. Bessa, D. F. Legendre and R. H. A. Prado (2007). Physiological pulsatile waveform through axisymmetric stenosed arteries: numerical simulation. ABCM Symposium Series, Rio de Janeiro.

O'Rourke, M. J., J. P. McCullough and S. Kelly (2012) An investigation of the relationship between hemodynamics and thrombus deposition within patient-specific models of abdominal aortic aneurysm. Proceedings of the Institution of Mechanical Engineers, Part H: Journal of Engineering in Medicine. 226(7):548-564

Parker, L. P., J. T. Powell, L. J. Kelsey, B. Lim, R. Ashleigh, M. Venermo, I. Koncar, P. E. Norman and B. J. Doyle (2019). 
A. A. Soares et al. / JAFM, Vol. 14, No. 6, pp. 1657-1668, 2021.

Hemodynamics in Isolated Common Iliac Artery Aneurysms Impacts Proximal Aortic Remodeling. Arterioscler Thromb Vasc Biol. 1125-1136.

Peiffer, V., S. J. Sherwin and P. D. Weinberg (2013) Does low and oscillatory wall shear stress correlate spatially with early atherosclerosis? A systematic review. Cardiovascular Research 99, 242-250.

Rikhtegar, F., J. A. Knight, U. Olgac, S. C. Saur, D. Poulikakos, W. Marshall Jr, P. C. Cattin, H. Alkadhi and V. Kurtcuoglu (2012). Choosing the optimal wall shear parameter for the prediction of plaque location-A patient-specific computational study in human left coronary arteries. Atherosclerosis 221, 432-437.

Soares, A. A., S. Gonzaga, C. Oliveira, A. Simões and A. Rouboa (2017). Computational fluid dynamics in abdominal aorta bifurcation: nonNewtonian versus Newtonian blood flow in a real case study. Computer Methods in Biomechanics and Biomedical Engineering 20, $1-10$.

Soares, A. A., S. Gonzaga, J. S. Silva, D. Marinho, A. Silva and A. Rouboa (2011). NonNewtonian blood flow in portal vein with thrombosis: a study by Computational Fluid Dynamics. In American Institute of Physics Conference Series 1389, 171-174.

Soares, A. A., F. A. Carvalho and A. Leite (2021). Effects of inlet velocity profile on the hemodynamics of the abdominal aorta bifurcation. Journal of the Brazilian Society of Mechanical Sciences and Engineering 43, 282.

Soulis, J. V., D. K. Fytanidis, K. V. Seralidou and G. D. Giannoglou (2014). Wall shear stress oscillation and its gradient in the normal left coronary artery tree bifurcations. Hippokratia $18,12-16$

Tang, B. T., C. P. Cheng, M. T. Draney, N. M. Wilson, P. S. Tsao, R. J. Herfkens and C. A. Taylor (2006). Abdominal aortic hemodynamics in young healthy adults at rest and during lower limb exercise: quantification using image-based computer modeling. American Journal of Physiology. Heart and Circulatory Physiology 291(2), 668-676.

Taylor, C. A., T. J Hughes and C. K. Zarins (1998). Finite element modeling of three-dimensional pulsatile flow in the abdominal aorta: relevance to atherosclerosis. Annals of biomedical engineering 26 (6), 975-987.

Taylor, C. A. (1996). A computational framework for investigating hemodynamic vascular adaptation and disease. Stanford: Stanford University.

Wen, C. Y., A. S. Yang, L. Y. Tseng and J. W. Chai (2010). Investigation of pulsatile flow field in healthy thoracic aorta models. Annals of Biomedical Engineering 38(2), 391-402.

Wong, K. K. L., D. Wang D., J. K. L Ko, J. Mazumdar, T .T Le and D. Ghista (2017). Computational medical imaging and hemodynamics framework for functional analysis and assessment of cardiovascular structures. BioMed Eng OnLine 16, 35.

Youssefi, P., A. Gómez, C. J. Arthurs, R. Sharma, M. Jahangiri and C. A. Figueroa (2018). Impact of Patient-Specific Inflow Velocity Profile on Hemodynamics of the Thoracic Aorta. Journal of Biomechanical Engineering 140(1): 011002 (14 pages).

Xing, R., A. M. Moerman, Y. Ridwan, M. J. Daemen, A. F. W. van der Steen, F. J. H. Gijsen and K. van der Heiden (2018). Temporal and spatial changes in wall shear stress during atherosclerotic plaque progression in mice. Royal Society Open Science 5(3), 171447.

Zeng, Y. Q. and Z. Y. Li. (2012). A Follow up MRIbased geometry and computational fluid dynamics study of carotid bifurcation. World Congress on Medical Physics and Biomedical Engineering, Beijing, China. 